Published in final edited form as:

N Engl J Med. 2009 January 29; 360(5): 532-534. doi:10.1056/NEJMcibr0808487.

\title{
Getting to the Heart of Proteomics
}

\author{
Peipei Ping, Ph.D. \\ From the NHLBI Program on Myocardial Ischemia Injury and Protection at the David Geffen School \\ of Medicine, University of California at Los Angeles, CA
}

Che-Hong Chen and colleauges ${ }^{1}$ recently described the mitochondrial aldehyde dehydrogenase-2 (ALDH-2) as a target in protecting the heart against ischemic insult. Their study provides insights into cardiovascular biology and may form the basis of a potential therapeutic approach to prevent or to minimize myocardial ischemic injury in the clinical setting.

Cardiac cells have innate defense mechanisms against injury that results from prolonged disruption of blood flow consequent to heart attack. ${ }^{2-5}$ The presence of these mechanisms was originally unmasked with the discovery that brief episodes of ischemia and reperfusion protect the heart against a subsequent prolonged ischemic insult. This process is known as ischemic preconditioning. Central to preconditioning is the activation of a signaling molecule, the epsilon form of protein kinase $\mathrm{C}$ (PKC $\varepsilon$ ), which mobilizes an endogenous cardiac defense program against injury and thereby has an infarct-sparing effect ${ }^{3,5}$.

These discoveries have spurred intensive research efforts to find pharmacologic agents that recapitulate the effects of ischemic preconditioning. Nitrates, adenosine agonists and volatile anesthetics, shown to be cardioprotective in the laboratory, have raised the spectra of protecting patients from myocardial damage, particularly in clinical settings-such as transplant and bypass surgery-in which ischemia can be anticipated. That said, translating experimental cardioprotective strategies remains a major challenge.

Investigations into the underlying cellular mechanisms of ischemic injury increasingly point towards a predominant role for mitochondria. ${ }^{4}$ Mitochondria are not only the main energy producers of the cardiac cell; they are also also host to specific signaling pathways for biogenesis and programmed cell death. Myocardial ischemia leads to increased mitochondrial $\mathrm{Ca}^{2+}$ uptake, which in turn increases mitochondrial permeability (this is correct), leading to breakdown of mitochondrial respiration and ultimately cell death. During preconditioning, PKC $\varepsilon$ prevents this degenerative process ${ }^{3,5}$ (this is correct) by phosphorylating molecules that trigger a cascade of events that ultimately preserve mitochondrial function. 2,4

The mammalian mitochondrion contains over 1,500 different proteins; many of which are decorated with various types of post-translational modification. In contrast to evaluating single molecules one at a time, a proteomic analysis provides a holistic portrait of the dynamic changes in mitochondrial proteomes during ischemia/reperfusion and offers an effective means of identifying protein targets relevant to disease. The study by Chen et al. ${ }^{1}$ combines the power of proteomics with a "classic" animal model of myocardial infarction (see Figure). Using a rodent model of myocardial ischemic injury, the authors report that phosphorylation of mitochondrial ALDH2 in response to protective stimuli (such as PKC $\varepsilon$ activation or ethanol treatment) increased the enzyme's activity and reduced myocardial infarct size. Next, they identified Alda-1 as a specific ALDH2 agonist that activated different isoforms of ALDH2, including that encoded by $A L D H 2 * 2$, a common East Asian allele. When administered before ischemia in the setting of global ischemic injury, Alda-1 reduced infarct size, thereby acting 
as a preconditioning mimetic. Activation of ALDH2 by Alda-1 was dependent on mitochondrial $\mathrm{PKC} \varepsilon$.

There are many efforts to translate insights from studies of preconditioning to the clinical arena. One such effort is the search for new and potentially more specific molecular targets for drug development. The proteomic approach used by Chen et al. ${ }^{1}$ circumvented preconceived notions about what molecules are involved, and enabled the identification of a key molecular target of preconditioning. Coupling this approach with whole animal physiology studies and small molecule screening, they have provided a convincing and novel mechanism with potential clinical relevance. Although substantive further research is clearly necessary, ALDH2 activators may at some point protect patients against myocardial protection. Even limited restoration of ALDH2 activity by Alda-1 may be relevant to the prevention of myocardial infarction in those who carry the ALDH $2 * 2$ allele. Dr. Ping reports no relevant financial conflicts of interest.

\section{References}

1. Chen CH, Budas GR, Churchill EN, Disatnik MH, Hurley TD, Mochly-Rosen D. Activation of aldehyde dehydrogenase-2 reduces ischemic damage to the heart. Science 2008;321(5895):1493-5. [PubMed: 18787169]

2. Murphy E, Steenbergen C. Mechanisms underlying acute protection from cardiac ischemia-reperfusion injury. Physiol Rev 2008;88(2):581-609. [PubMed: 18391174]

3. Ping P, Zhang J, Qiu Y, et al. Ischemic preconditioning induces selective translocation of protein kinase $\mathrm{C}$ isoforms epsilon and eta in the heart of conscious rabbits without subcellular redistribution of total protein kinase C activity. Circ Res 1997;81(3):404-14. [PubMed: 9285643]

4. Weiss JN, Korge P, Honda HM, Ping P. Role of the mitochondrial permeability transition in myocardial disease. Circ Res 2003;93(4):292-301. [PubMed: 12933700]

5. Ytrehus K, Liu Y, Downey JM. Preconditioning protects ischemic rabbit heart by protein kinase C activation. Am J Physiol 1994;266(3 Pt 2):H1145-52. [PubMed: 8160817] 


\section{Rodent Experimental Model}

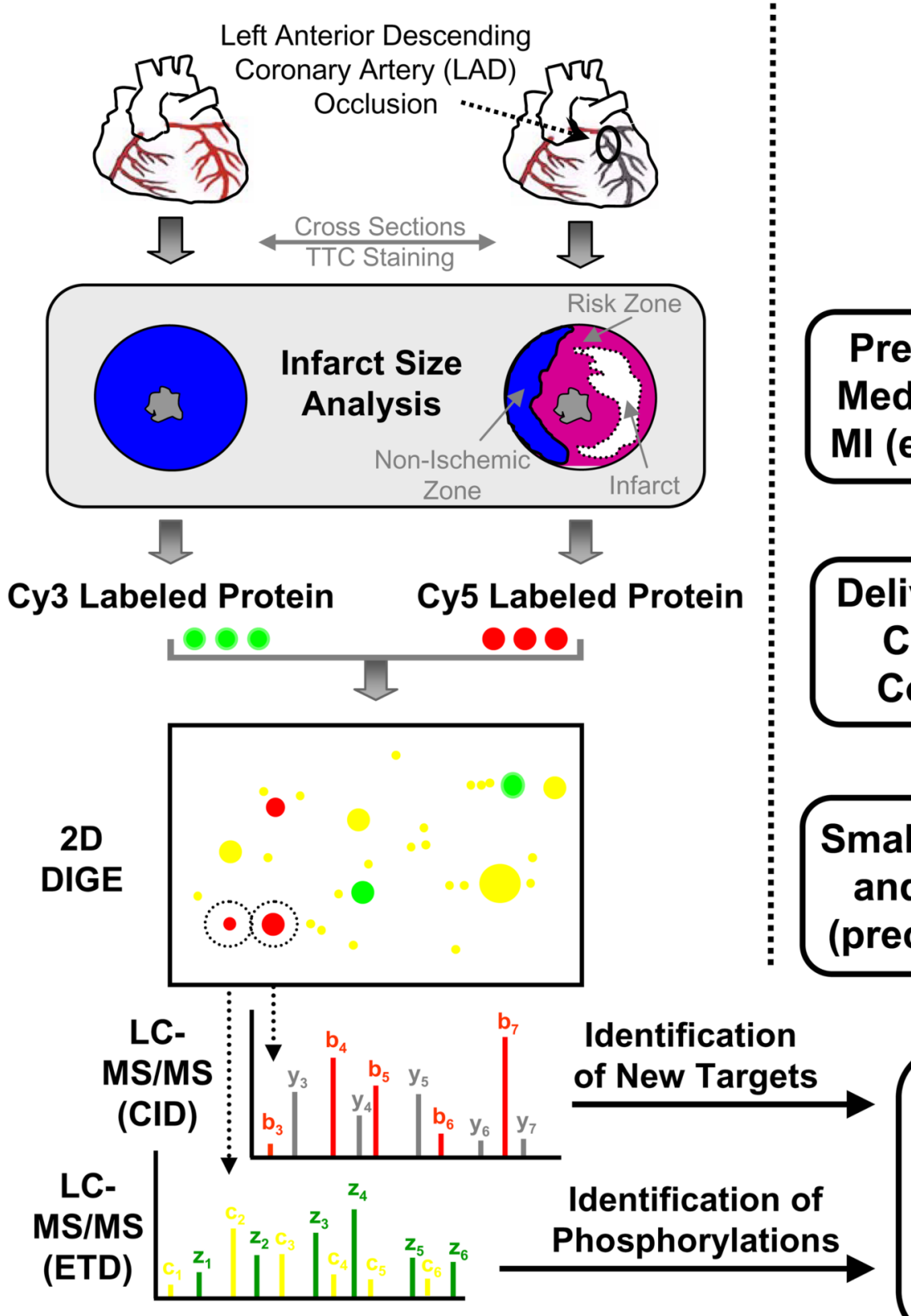

\section{Clinical Translation}

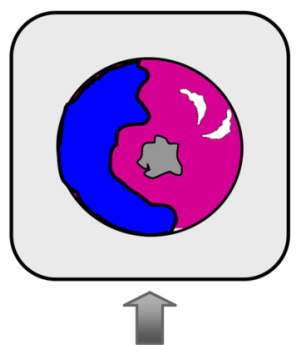

Preconditioning Based Medications to Minimize MI (e.g. Alda-1 or mimics)

Delivery of Therapies to Cases Anticipating Coronary Occlusion

Figure 1. The Power of Proteomics

Chen et al. ${ }^{1}$ recently analyzed, by using a proteomic approach, experimental heart injury models. They extracted protein content from sham and injured hearts and then labeled the proteins with fluorescent dyes (e.g. Cy3 in green, Cy5 in red). They then mixed the samples and resolved them on a two-dimensional gel according to isoelectric point and molecular weight (e.g. 2D DIGE). Protein spots that are identical between the sham and injured hearts are shown as yellow; protein spots that are more abundant in the sham hearts are shown as green; whereas red protein spots indicate a higher abundance in the injured hearts. Protein spots of interest were excised from the gel and subjected to analysis by liquid chromatography mass spectrometry (LC-MS/MS). Mass spectrometers set for collision induced dissociation (CID) 
are preferable for protein identification, whereas those set for electron transfer dissociation (ETD) are preferable for the identification of phosphorylation sites. Target candidate proteins such as ALDH2, are then validated and screened for the development of potential therapeutics. 